TITLE:

\title{
Verifying fault release control of power system via hybrid system reachability
}

\section{$\operatorname{AUTHOR}(\mathrm{S}):$}

Susuki, Yoshihiko; Sakiyama, Takuya; Ochi, Takashi; Uemura, Takuji; Hikihara, Takashi

\section{CITATION:}

Susuki, Yoshihiko ... [et al]. Verifying fault release control of power system via hybrid system reachability. 40th North American Power Symposium (NAPS2008) 2008: 5307297.

ISSUE DATE:

2008-09

URL:

http://hdl.handle.net/2433/66188

RIGHT: 


\section{Verifying Fault Release Control of Power System via Hybrid System Reachability}

\author{
Yoshihiko Susuki \\ Kyoto University \\ Department of Electrical Engineering \\ Katsura, Nishikyo, Kyoto 615-8510 \\ JAPAN \\ susukilieee.org
}

\author{
Takuya Sakiyama \\ Takashi Ochi \\ Takuji Uemura \\ Kansai Electric Power Co., Inc. \\ Power Engineering R\&D Center \\ Nakoji, Amagasaki, Hyogo 661-0974
}

\author{
Takashi Hikihara \\ Kyoto University \\ Department of Electrical Engineering \\ Katsura, Nishikyo, Kyoto 615-8510 \\ JAPAN
}

\begin{abstract}
Hybrid system reachability is applied to verification of fault release control in a power system. Fault release control is one strategy in emergency control and aims to mitigate an electrical stress of power system caused by disturbances. We introduce practical data in a fault release control of double machine-infinite bus (DMIB) system. The data is obtained using the RTDS and describes that the control is effective for prevention of transient instability in the DMIB system. By modeling of swing dynamics of the DMIB system as a hybrid automaton and reachability analysis of the automaton, we show that the control is correct for the prevention of transient instability.
\end{abstract}

\section{INTRODUCTION}

Power system is a safety-critical engineered system supplying electrical energy. The malfunction of power system causes damage to human activity, and this was proved in fact by the 2003 blackouts in North America and Europe. Verification of power system [1] is then needed for guaranteeing its stability and reliability. Many current problems of power systems are formulated as verification ones. Examples are occurrence of cascading outages and blackouts, penetration of distributed power sources, and development of electricity markets.

We proposed in [2], [3] to use hybrid system reachability for stability analysis of power systems. Hybrid systems are dynamical systems interacting with continuous- and discretevalued states [4]. Hybrid system reachability is widely used for verification of safety properties of system whose behaviors are represented by a mathematical model of hybrid system [5], [6], [7]. Power system dynamics and control contain hybrid nature [8], [9], [10], implying that they are regarded as dynamic interaction between continuous behaviors of frequency or voltage and discrete controls such as protective relay operation. It is therefore said that power system control is a typical application of safety verification for hybrid systems [1]. The method proposed in [2], [3] consists of two procedures: $(A)$ modeling of power system dynamics using hybrid automaton $[11]$ and $(B)$ reachability analysis of the automaton.

The purpose of this paper is to apply hybrid system reachability to verification of fault release control in a power system. Fault release control is one strategy in emergency control and aims to mitigate an electrical stress of power system caused by disturbances. The control is implemented by discontinuous change of power system topology and is hence regarded as a hybrid control. We introduce practical data in a fault release control of double machine-infinite bus (DMIB) system. The data is obtained using the RTDS (Real Time Digital Simulator) and shows that the control is effective for prevention of transient instability in the DMIB system. We next model as a hybrid automaton the interaction of coupled swings in two generators and of fault release control. By reachability analysis of the automaton, we show that the fault release control is correct for the prevention of transient instability. The contribution of this paper is to show that hybrid system reachability is applicable to verification of fault release control. Preliminary discussions were reported in [12], [13], [14].

\section{HybRid System REACHABILITY}

There are many papers about theory of modeling, analysis, and control of hybrid systems. This section presents only two concepts on hybrid systems theory: hybrid automaton and its reachability. The contents below are based on [15].

\section{A. Hybrid Automaton}

Hybrid automaton is a well-known mathematical model of hybrid systems, and it has been analyzed theoretically and used in various applications. A form of hybrid automaton, denoted by $\mathrm{H}$, is given by

$$
\mathrm{H}=(\mathbb{Q}, \mathbb{X}, U, f, \text { Dom, } G, r),
$$

with

- $\mathbb{Q}$ is a finite set of discrete states. $X$ is a set of continuous states. A pair $(q, x) \in \mathbb{Q} \times \mathbb{X}$ is the state of $\mathrm{H}$;

- $U=\left(U_{\mathrm{C}}, U_{\mathrm{D}}\right)$ is a set of continuous and discrete inputs;

- $f: \mathbb{Q} \times \mathbb{X} \times U_{\mathrm{C}} \rightarrow \mathrm{T} \mathbb{X}$ is a function which describes continuous dynamics (vector field). $T \mathbb{X}$ is the tangent bundle of $\mathbb{X}$;

- Dom : $\mathbb{Q} \times U_{\mathrm{D}} \rightarrow P(\mathbb{X})$ is a domain of continuous dynamics and describes a subset of $\mathbb{X}$ within which continuous dynamics are permitted. $P(\mathbb{X})$ is the power set of $\mathbb{X}$; 


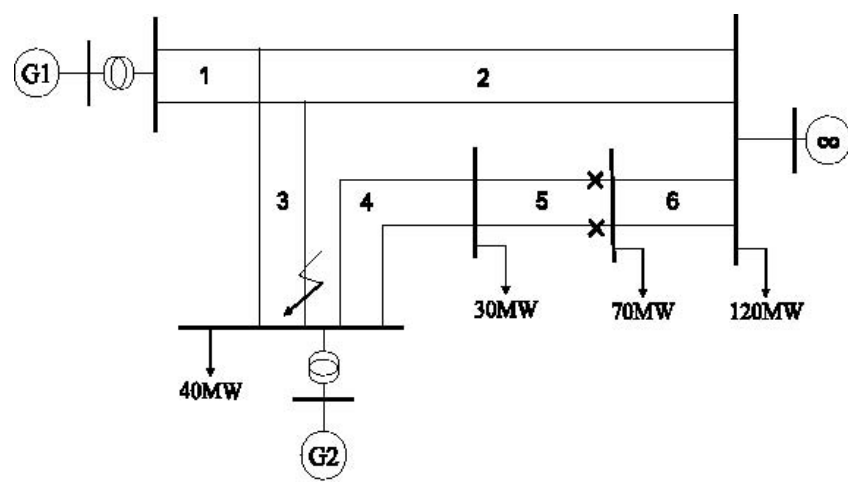

Fig. 1. Double machine-infinite bus system

- $G: \mathbb{Q} \times \mathbb{Q} \times U_{\mathrm{D}} \rightarrow P(\mathbb{X})$ is called guard condition for discrete transition and describes transition relations of discrete states;

- $r: \mathbb{Q} \times \mathbb{Q} \times \mathbb{X} \times U \rightarrow \mathbb{X}$ is called reset function and describes changes of continuous states at discrete transition.

Trajectories of $\mathrm{H}$ evolve continuously as well as in discrete jumps. Mathematical descriptions of trajectories and semantics of $\mathrm{H}$ are presented in [7] and references therein. $U_{\mathrm{C}}$ is not used in this paper.

\section{B. Reachability}

Reachability analysis of $\mathrm{H}$ seeks to determine whether trajectories of $\mathrm{H}$ can reach a subset of states, denoted by target set $T$, from a subset of initial states, denoted by $I$. Reachability analysis is used for verification of safety properties of system whose behaviors are modeled by $\mathrm{H}$. T is then fixed as a subset of states that describe undesirable or unsafe behaviors of the system, called unsafe set. There are two types of analysis. Forward reachability starts with states in $I$ and follows trajectories forward in time. If any of forward trajectories intersect with $T$, the system is unsafe. Backward reachability starts with states in $T$ and follows trajectories backward in time. If any of backward trajectories intersects with $I$, the system is unsafe. The set of forward (or backward) trajectories are called forward (or backward) reach set ${ }^{1}$.

\section{NumericAl Experiment of FAult Release CONTROL}

This section introduces practical data of a fault release control of double machine-infinite bus (DMIB) system. From the data we formulate one verification problem of fault release control. This problem is solved in Sec. IV using hybrid system reachability.

\section{A. System Model and Protection Control}

The DMIB system is shown in Fig. 1 and consists of two synchronous generators, infinite bus, ac transmission network, loads, relays, and circuit breakers. Two synchronous

\footnotetext{
${ }^{1}$ Forward (or backward) reach set coincides with forward (or backward) reach tube in [15].
}

generators, denoted by G1 and G2, are coupled via the ac transmission network. Arrows in the figure denote constant impedance loads. The DMIB system has the three protection systems, each of which consists of relay and circuit breaker. Two of them are equipped with G1 and G2, and the other is equipped with line 5. Control mechanisms for the protection systems are as follows:

- Generation trip: The protection system with generators consists of the impedance-type relay and circuit breaker, and aims to remove G1 and G2 from the ac transmission network when they start to show transient instability. The operation is based on complex net impedance of the network measured from each generator bus. The breaker trips to remove generators from the network if the net impedance passes the imaginary axis from right domain of complex plane to left. The protection system is regarded as a controller with single continuous-valued input, namely, the net impedance, and single discretevalued output.

- Fault release: The protection system with line 5 is for fault release control and aims to mitigate an electrical stress caused by disturbances. The circuit breaker with the protection system trips to disconnect the line a prescribed period (normally, a few cycles) after either generator is removed by the above protection system. That is, the onset of line disconnection depends on that of generation trip. The protection system is also regarded as a controller with single discrete-valued output. Sec IV fixes a more specified objective of the fault release control in order to use hybrid system reachability.

The numerical experiment here is performed using the RTDS (Real Time Digital Simulator) produced by RTDS Technologies Inc. The RTDS is a fully digital electromagnetic transient power system simulator. Generator dynamics are modeled by the two-axis Park equations with damper windings. The effects of AVR and PSS can be considered; however, they are assumed not to operate in this paper. The fault setting is as follows: Suppose that the DMIB system is at a steady operating condition before $t<t_{\mathrm{f}}=0 \mathrm{~s}$, that a three phase lines-to-ground fault occurs near G2 bus and at $t=t_{\mathrm{f}}=0 \mathrm{~s}$, and that the faulted line is tripped at $t=t_{\mathrm{cl}}=8 /(60 \mathrm{~Hz}) \sim 0.133 \mathrm{~s}$, i.e., 8 cycles of $60 \mathrm{~Hz}$ sine wave. The fault release control is assumed to occur $0.3 \mathrm{~s}$ after either generator is removed by the protection system. System parameters including generator and line data are shown in Tab. I.

\section{B. Practical Data}

Figure 2 shows transient behaviors of rotor speed deviation $\omega_{1}$ and $\omega_{2}$ relative to nominal angular frequency $(2 \pi \times 60 \mathrm{~Hz})$ : (a) for G1 and (b) for G2. Fig. 2(a) describes two behaviors denoted by Case 1 and Case2. Case1 represents the time response without fault release control. Case2, on the other hand, represents the response with it. The onset of control is denoted by CB-in (line5). G2-off in Figs. 2(a) and (b) 
TABLE I

PARAMETERS OF DOUBLE MACHINE-INFINITE BUS SYSTEM (a) Line data (10 MVA base per 1-line)

\begin{tabular}{ccr}
\hline No. & $\% Z=R+\mathrm{j} X$ & $\mathrm{j} Y$ \\
\hline 1 & $0.14+\mathrm{j} 0.657$ & $\mathrm{j} 1.06$ \\
\hline 2 & $0.55+\mathrm{j} 2.55$ & $\mathrm{j} 3.70$ \\
\hline 3 & $0.73+\mathrm{j} 3.40$ & $\mathrm{j} 4.94$ \\
\hline 4 & $0.019+\mathrm{j} 0.037$ & $\mathrm{j} 5.38$ \\
\hline 5 & $0.028+\mathrm{j} 0.040$ & $\mathrm{j} 15.02$ \\
\hline 6 & $0.066+\mathrm{j} 0.252$ & $\mathrm{j} 41.28$ \\
\hline
\end{tabular}

(b) Transformer data (10 MVA base)

\begin{tabular}{rrrr}
\hline & Rated capacity & Rated voltage & $\% X$ \\
\hline G1 & $82 \mathrm{MVA}$ & $13.8 \mathrm{kV} / 77.0 \mathrm{kV}$ & $\mathrm{j} 0.967$ \\
\hline $\mathrm{G} 2$ & $160 \mathrm{MVA}$ & $15.0 \mathrm{kV} / 77.0 \mathrm{kV}$ & $\mathrm{j} 0.618$ \\
\hline
\end{tabular}

(c) Generator data (Rated capacity base)

\begin{tabular}{cccccr}
\hline & $\begin{array}{c}\text { Rated } \\
\text { capacity }\end{array}$ & $\begin{array}{c}\text { Rated } \\
\text { voltage }\end{array}$ & $\begin{array}{c}\text { Real } \\
\text { power }\end{array}$ & $\% X_{d}^{\prime}$ & \multicolumn{1}{c}{$\begin{array}{c}\text { inertia } \\
\text { constant }(M)\end{array}$} \\
\hline G1 & $81.176 \mathrm{MVA}$ & $13.8 \mathrm{kV}$ & $70 \mathrm{MW}$ & $\mathrm{j} 26.1$ & $10.0 \mathrm{~s}$ \\
\hline G2 & $160 \mathrm{MVA}$ & $15.0 \mathrm{kV}$ & $125 \mathrm{MW}$ & $\mathrm{j} 28.4$ & $6.88 \mathrm{~s}$ \\
\hline
\end{tabular}

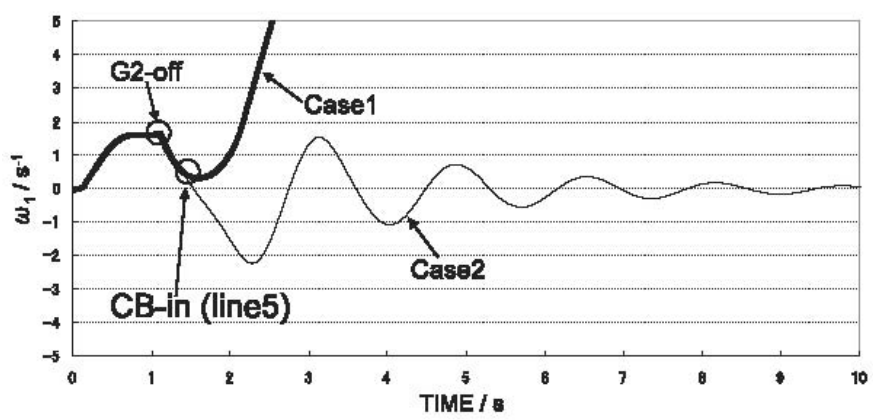

(a) Rotor speed deviation $\omega_{1}$ of $\mathrm{G} 1$

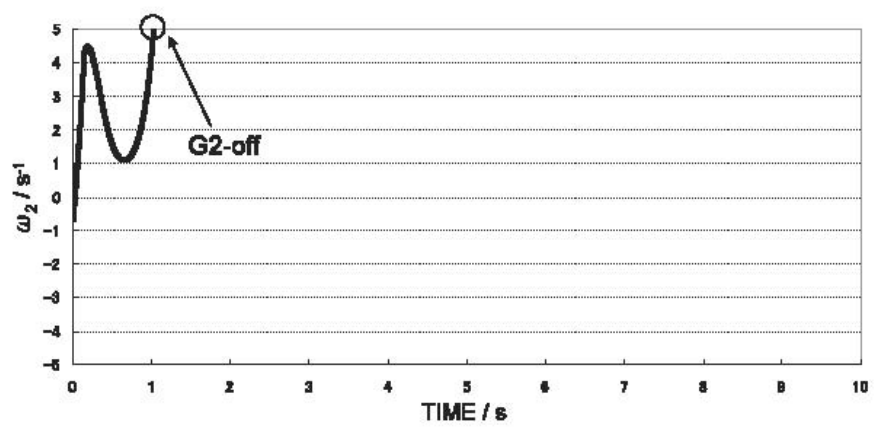

(b) Rotor speed deviation $\omega_{2}$ of $\mathrm{G} 2$

Fig. 2. Numerical experiment of fault release control of double machineinfinite bus system

also denotes the onset of G2 trip caused by the impedancetype protection. Fig. 2(b) describes that G2 shows divergence motion of $\omega_{2}$, that is, transient instability, not depending on the fault release control. Fig. 2(a), on the other hand, describes that G1 does not show divergence motion of $\omega_{1}$ under the fault release control and settles down a steady operating condition as time passes. These figures indicate that transient instability of G1 can be avoided by the fault release control. The next section verifies the correctness of fault release control using hybrid system reachability.

\section{Verification of Fault Release Control}

Section III showed through numerical experiment that transient instability of G1 can be avoided by the fault release control. This section uses hybrid system reachability for verifying the correctness of fault release control. The contents below consist of two parts: $(A)$ modeling of the interaction between continuous coupled swings and discrete controls as a hybrid automaton and $(B)$ reachability analysis of the automaton.

\section{A. Hybrid Modeling}

First we model swing dynamics of generators G1 and G2 that are the continuous dynamics $f$ in $\mathrm{H}$. Suppose that $\delta_{i}$ is the rotor position of $\mathrm{G} i$ with respect to the infinite bus, and $\omega_{i}$ is the rotor speed deviation of $\mathrm{G} i$ relative to the nominal angular frequency $\omega_{\mathrm{s}}=2 \pi \times(60 \mathrm{~Hz})$, then swing dynamics of the generators are modeled by the so-called classical swing equations:

$$
\left\{\begin{aligned}
\dot{\delta}_{1}= & \omega_{1} \\
a \frac{2 H_{1}}{\omega_{\mathrm{s}}} \dot{\omega}_{1}= & P_{\mathrm{m} 1}-D \omega_{1}-G_{11} V_{1}^{2} \\
& -\sum_{i=2, \infty}\left\{G_{1 i} V_{1} V_{i} \cos \left(\delta_{1}-\delta_{i}\right)\right. \\
\dot{\delta}_{2} & \left.\omega_{2}, \quad B_{1 i} V_{1} V_{i} \sin \left(\delta_{1}-\delta_{i}\right)\right\} \\
\frac{2 H_{2}}{\omega_{\mathrm{s}}} \dot{\omega}_{2}= & P_{\mathrm{m} 2}-D \omega_{2}-G_{22} V_{2}^{2} \\
& -\sum_{i=1, \infty}\left\{G_{2 i} V_{2} V_{i} \cos \left(\delta_{2}-\delta_{i}\right)\right. \\
& \left.\quad+B_{2 i} V_{2} V_{i} \sin \left(\delta_{2}-\delta_{i}\right)\right\}
\end{aligned}\right.
$$

$\dot{\delta}_{1}$ denotes the time differentiation of $\delta_{1} . \delta_{\infty}$ is the rotor position of infinite bus and is assumed to be zero. The parameters $a, H_{i}, P_{\mathrm{m} i}, D, G_{i i}, G_{i j}, B_{i j}$, and $V_{i}$ are in per unit system except for $H_{i}$ and $D$ in second. $a$ is the ration of generator rated capacity of $\mathrm{G} 1$ to that of $\mathrm{G} 2, H_{i}$ the per unit inertia constant of $\mathrm{G} i$, and $D$ the damping coefficient of generators. $G_{i i}$ is the internal impedance, and $G_{i j}+\mathrm{j} B_{i j}$ the transfer admittance. $V_{1}$ and $V_{2}$ are the bus voltages of $\mathrm{G} 1$ and $\mathrm{G} 2$, and $V_{\infty}$ the voltage of infinite bus.

Second we model the protection control of generation trip and fault release. The modeling procedure below is similar to that in [16]. The parameters $G_{i i}, G_{i j}$, and $B_{i j}$ in equation (2) discontinuously change when the fault, generation trip, and line trip occurs by the protection control. For hybrid modeling, it is needed to describe candidates of network topologies explicitly. The data in Sec. III-B requires the following discrete set $\mathbb{Q}$ in order to describe it as

$$
\mathbb{Q}=\left\{q_{1}, q_{2}, q_{3}, q_{4}\right\} .
$$

The discrete state $q_{1}$ is the system condition for fault-on state. $q_{2}$ is the condition after removing the faulted line, $q_{3}$ the condition after removing $\mathrm{G} 2$ by generation trip relay, and $q_{4}$ the condition after fault release control. Note that other discrete states are needed to describe all the candidates of network topologies. Here we re-write equation (2) to consider the 
network topologies. The electrical output term $P_{\mathrm{e} i}^{(q)}$ indexed by $q \in \mathbb{Q}$, which represents the third and fourth terms in righthand side of equation (2), is given by

$$
\begin{array}{r}
P_{\mathrm{e} i}^{(q)}=G_{i i}^{(q)} V_{i}^{2} \\
+\sum_{j=1,2, \infty, j \neq i}\left\{G_{i j}^{(q)} V_{i} V_{j} \cos \left(\delta_{i}-\delta_{j}\right)\right. \\
\left.+B_{i j}^{(q)} V_{i} V_{j} \sin \left(\delta_{i}-\delta_{j}\right)\right\},
\end{array}
$$

where $i=1,2$. The parameter $G_{i i}^{(q)}$ denotes the internal impedance of $\mathrm{G} i$ for index $q$, and similarly for $G_{i j}^{(q)}, B_{i j}^{(q)}$. The generation trip and fault release themselves are represented by members of discrete control $U_{\mathrm{D}}$. For the data in Sec. III-B, the protection control which removes the faulted line is modeled by the discrete control $\sigma^{1}$, and it drives the discrete transition from $q_{1}$ to $q_{2}$. The protection control which removes $\mathrm{G} 2$ is modeled by $\sigma^{2}$, and it drives the discrete transition from $q_{2}$ to $q_{3}$. The fault release control is also denoted by $\sigma^{3}$, and it drives the discrete transition from $q_{3}$ and $q_{4}$.

Last we integrate the continuous dynamics in equation (2) with the discrete transition above and obtain a hybrid automaton used for the verification problem. $\mathrm{H}$ is given as follows:

$$
\left\{\begin{array}{l}
\mathbb{Q}=\left\{q_{1}, q_{2}, q_{3}, q_{4}\right\}, \\
\mathbb{X}=S^{2} \times \mathbb{R}^{2} \times \mathbb{R}^{+} \ni x=\left(\delta_{1}, \delta_{2}, \omega_{1}, \omega_{2}, z\right), \\
U_{\mathrm{C}}=\emptyset \\
U_{\mathrm{D}}=\left\{\sigma^{1}, \sigma^{2}, \sigma^{3}\right\} \\
f\left(q_{i}, x\right), q_{i} \in \mathbb{Q}, i=1,2,3,4, \\
D o m=\mathbb{Q} \times \mathbb{X}, \\
G\left(q_{i}, q_{i+1}, x, \sigma^{i}\right), i=1,2,3, \\
r\left(q_{i}, q_{i+1}, x, \sigma^{i}\right)=x, i=1,2,3 .
\end{array}\right.
$$

A schematic diagram of $\mathrm{H}$ is in Fig. 3. The new variable $z / \mathrm{s} \in \mathbb{R}^{+}$with continuous dynamics $\dot{z}=1$ is added to $\mathbf{X}$ in order to drive the discrete controls $\sigma^{1}$ and $\sigma^{3}$. The continuous dynamics $f$ are then given by

$$
f\left(q_{i}, x\right)=\left(\begin{array}{c}
\omega_{1} \\
\omega_{2} \\
\frac{\omega_{\mathrm{s}}}{2 H_{1} a}\left\{P_{\mathrm{m} 1}-D \omega_{1}-P_{\mathrm{e} 1}^{\left(q_{i}\right)}(x)\right\} \\
\frac{\omega_{\mathrm{s}}}{2 H_{2}}\left\{P_{\mathrm{m} 2}-D \omega_{2}-P_{\mathrm{e} 2}^{\left(q_{i}\right)}(x)\right\} \\
1
\end{array}\right) .
$$

The guard condition $G$ is based on the control mechanisms of the protection systems and is defined as

$$
\left\{\begin{array}{l}
G\left(q_{1}, q_{2}, x, \sigma^{1}\right)=\left\{x \in \mathbb{X} \mid z=t_{\mathrm{cl}}\right\}, \\
G\left(q_{2}, q_{3}, x, \sigma^{2}\right) \\
=\left\{x \in \mathbb{X} \mid \operatorname{Re}\left[Z_{2}(x)\right]=0\right. \\
\left.\quad \text { and } \mathrm{d}\left(\operatorname{Re}\left[Z_{2}(x)\right]\right) / \mathrm{d} t<0\right\}, \\
G\left(q_{3}, q_{4}, x, \sigma^{3}\right)=\left\{x \in \mathbb{X} \mid z=t_{2}+\Delta t\right\},
\end{array}\right.
$$

where $Z_{2}(x)$ is the complex net impedance of ac transmission network measured from $\mathrm{G} 2$ bus, and its real part is represented

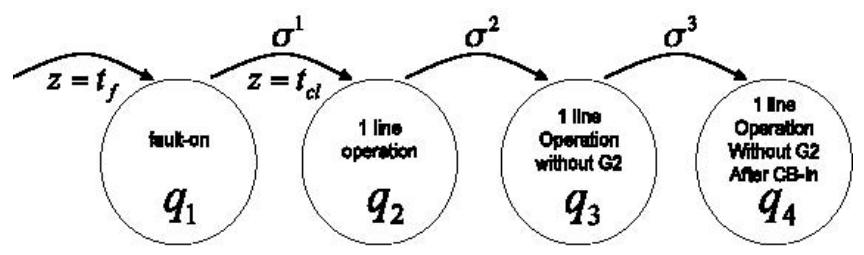

Fig. 3. Schematic diagram of hybrid automaton $\mathrm{H}$ showing the interaction between coupled swings of the generators and discrete controls for the protection systems

by

$$
\begin{aligned}
\operatorname{Re}\left[Z_{2}\right] & =\frac{V_{2}}{\left|I_{2}^{\left(q_{2}\right)}\right|^{2}} \cdot \\
\cdot & \left\{\cos \delta_{2} \sum_{j=1,2, \infty} V_{j}\left(G_{2 j}^{\left(q_{2}\right)} \cos \delta_{j}-B_{2 j}^{\left(q_{2}\right)} \sin \delta_{j}\right)\right. \\
& \left.+\sin \delta_{2} \sum_{j=1,2, \infty} V_{j}\left(G_{2 j}^{\left(q_{2}\right)} \sin \delta_{j}+B_{2 j}^{\left(q_{2}\right)} \cos \delta_{j}\right)\right\} .
\end{aligned}
$$

$I_{2}^{\left(q_{2}\right)}$ is the complex output current of $\mathrm{G} 2$ that is also a function of rotor positions $\left(\delta_{1}, \delta_{2}\right)$ and discrete variable $q_{i}$. For $G\left(q_{3}, q_{4}, x, \sigma^{3}\right)$ in equation (7), $t_{2}$ is the onset time of discrete control $\sigma^{2}$, and $\Delta t$ is the prescribed delay time for the fault release control. The reset condition $r$ in equation (5) implies that there is no jump of continuous evolution at the onset of discrete transitions.

\section{B. Reachability Analysis}

We use forward reachability of $\mathrm{H}$ for verifying the correctness of fault release control. To explore forward reach sets, it is needed to fix the unsafe set $T$ and the initial set $I$. The setting of $T$ depends on the objective of controller/algorithm whose performance is verified. We now regard the objective of fault release control as avoiding transient instability of G1 and define the unsafe set $T$ as

$$
T=\left\{(q, x) \in \mathbb{Q} \times \mathbb{X} \mid \omega_{1} \geq \omega_{\mathrm{cr}}\right\}
$$

where $\omega_{\mathrm{cr}}(>0 \mathrm{rad} / \mathrm{s})$ is the critical upper value of $\omega_{1}$ which the generator can operate safely. The reason why $\omega_{\text {cr }}$ is used is that if G2 is removed from the network, transient instability of G1 is well represented by a solution converging to the stable periodic solution of the second kind, positioned at $\omega_{1}(t) \sim P_{\mathrm{m} 1} / D(>0)$, in equation (2). On the other hand, the setting of $I$ depends on how initial operating conditions of the DMIB system are assumed. The data in Sec. III-B is obtained under the assumption at $t=t_{\mathrm{f}}$ that the DMIB system is at a steady operating condition. With these in our mind, we use the following setting of $I$ :

$$
\begin{aligned}
I= & \left\{(q, x) \in \mathbb{Q} \times \mathbb{X} \mid z=t_{\mathrm{f}},-\pi \leq \delta_{1}<\pi,\right. \\
& -5 \mathrm{rad} / \mathrm{s} \leq \omega_{1} \leq 5 \mathrm{rad} / \mathrm{s}, \delta_{2}=\delta_{2}^{*}, \\
& \left.\omega_{2}=0 \mathrm{rad} / \mathrm{s}\right\},
\end{aligned}
$$


TABLE II

Parameter Setting of Hybrid Automaton H

(a) Fixed values

\begin{tabular}{cccccc}
\hline$H_{1}$ & $5 \mathrm{~s}$ & $H_{2}$ & $3.5 \mathrm{~s}$ & $\omega_{\mathrm{s}}$ & $2 \pi \times(60 \mathrm{~Hz})$ \\
\hline$P_{\mathrm{m} 1}$ & 0.28 & $P_{\mathrm{m} 2}$ & 0.75 & $V_{i}$ & 1 \\
\hline$D$ & $0.01 \mathrm{~s}$ & $t_{\mathrm{f}}$ & $0 \mathrm{~s}$ & $t_{\mathrm{cr}}$ & $0.133 \mathrm{~s}$ \\
\hline$\Delta t$ & $0.3 \mathrm{~s}$ & $\omega_{\mathrm{cr}}$ & $5 \mathrm{rad} / \mathrm{s}$ & & \\
\hline
\end{tabular}

(b) Internal and transfer admittance $G_{i j}^{(q)}+\mathrm{j} B_{i j}^{(q)}$ Pre-fault topology

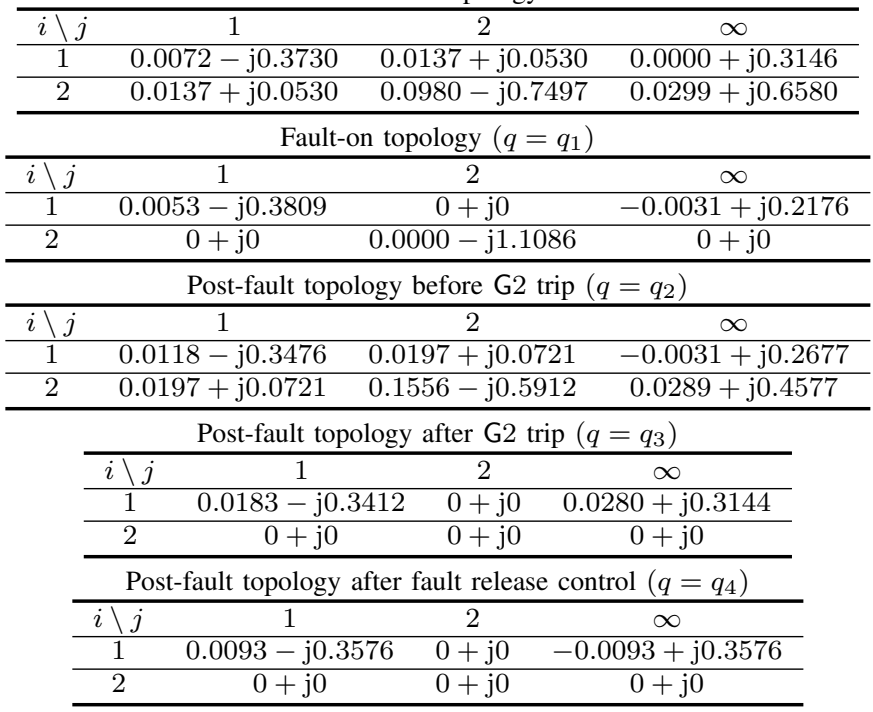

where $\delta_{2}^{*}$ is the value at the steady operating condition, in this case, a stable equilibrium point. $I$ contains the stable point of equation (2) before the fault. The reason why $I$ does has non-zero measure is that we attempt to analyze the correctness of fault release control with considering perturbations to the initial conditions.

There are many contributions to theory of reachability analysis of hybrid systems: see references in [15]. Estimation methods for forward reachability of hybrid systems with nonlinear dynamics by reduction to linear problems are proposed in [17], [18], which are called Lagrangian approach. We perform the reachability analysis by direct numerical integration of equation (2). Namely, the set $I$ of initial states are finely partitioned by a grid, and the trajectory starting from each element of the grid is computed by numerical integration and is checked whether it intersects with $T$ or not. The parameter setting for numerical simulations is given in Tab. II.

Figure 4 shows numerical results of reachability analysis. Each bounded two-dimensional plane describes the set $I$ of initial conditions. The colored regions represent forward reach sets for the unsafe set $T$. Precisely, each region is a finite set of elements of the grid from which trajectory of $\mathrm{H}$ intersects with $T$ in time $5 \mathrm{~s}$. The non-colored (white) regions represent the subset of $I$ from which trajectory does not intersect with $T$ for $5 \mathrm{~s}$. Fig. 4(a) is the case without fault release control, namely, without the discrete control $\sigma^{3}$. The colored region is divided into three subsets. The subset denoted by $q_{i}$ is an approximation of the subset of $I$ from which trajectory of $\mathrm{H}$ goes to $T$ at discrete state $q_{i}$. IP denotes a steady operating

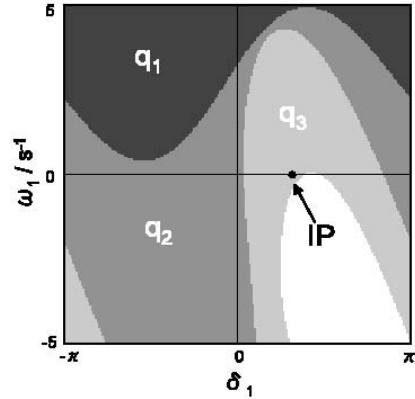

(a) Case1

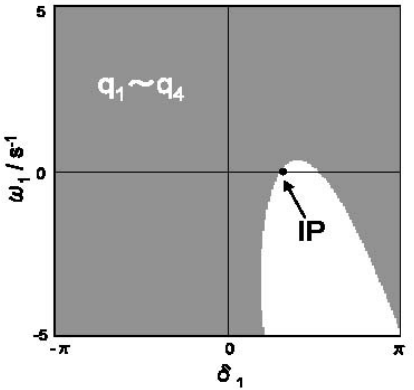

(b) Case2
Fig. 4. Numerical results of reachability analysis. Colored regions provide approximations of forward reach sets for the unsafe set $T$.

condition and corresponds to the initial state used for the RTDS in Sec. III-B. IP in Fig. 4(a) exists inside the colored region, that is, the forward reach set for $T$. This implies that as time passes, G1 shows transient instability without fault release control. This is consistent with the data in Fig. 2(a). On the other hand, Fig. 4(b) is the case with the fault release control $\sigma^{3}$. The colored region, denoted by $q_{1} \sim q_{3}$, represents an approximation the subset of $I$ from which trajectory intersects with $T$ at $q_{i}(i=1,2,3)$ in $5 \mathrm{~s}$. IP also denotes the initial state used for the RTDS and exists outside the colored region, i.e., the forward reach set. This implies that G1 does not show transient instability in $5 \mathrm{~s}$ with considering the fault release control. This is consistent with the data in Fig. 2(a). Thus the reachability analysis of hybrid automaton can verify that the fault release control is correct for prevention of transient instability of G1 caused by the fault near G2 bus.

\section{CONClusion}

We applied hybrid system reachability to verification of fault release control in a power system. This paper introduced practical data of a fault release control in double machineinfinite bus (DMIB) system, which was obtained by the RTDS (Real Time Digital Simulator). The dynamics related to the fault release control were modeled as a hybrid automaton and were analyzed via forward reachability of the automaton. Thus we show that the reachability analysis of hybrid automaton can verify the correctness of fault release control obtained by the RTDS.

Future work related to this paper is discussed. The present application of reachability analysis relies on numerical integration of continuous dynamics with considering discrete transitions. No theoretical result is provided which supports the reachability analysis of infinitely many initial states through a finite number of iterations of an algorithm. We are firmly of the opinion that such numerical results cannot prove something about safety verification problems. Here it is the fact that transient dynamics of power systems require high-dimensional nonlinear differential or differential-algebraic equations for their precise analyses [19]. Hence, although the present result on reachability is in a good agreement with the practical data, we need to use the estimation methods [17], [18] for 
reachability analysis of nonlinear hybrid systems, which is in future work. Another work is to use the obtained results of reachability analysis for designing and modifying control mechanisms of the protection systems.

\section{ACKNOWLEDGMENTS}

We are grateful to Mr. Hiroaki Ebina (Kyoto University), Mr. Kosaku Yokota (Kansai Electric Power Co., Inc.), Mr. Yu Takatsuji (Kyoto University), and Mr. Osamu Yatsubo (Kansai Electric Power Co., Inc.) for valuable discussions. We are also grateful to $\mathrm{Mr}$. Yu Takatsuji for his careful reading of the manuscript.

\section{REFERENCES}

[1] S. N. Talukdar, J. Apt, M. Ilic, L. B. Lave, and M. G. Morgan, "Cascading failures: Survival versus prevention," The Electricity Journal, vol. 16, no. 9, pp. 25-31, November 2003.

[2] T. Hikihara, "Application of hybrid system theory to power system analysis (I)," in Annual Meeting Record I.E.E.Japan, vol. 6, March 2005, p. 187, (in Japanese).

[3] Y. Susuki, H. Ebina, and T. Hikihara, "Application of hybrid system theory to power system stability analysis," in Proceedings of the 2005 International Symposium on Nonlinear Theory and its Applications, Bruge, Belgium, October 2005, pp. 202-205.

[4] M. Domenica, D. Benedetto, and A. Sangiovanni-Vincentelli, Eds., $H y-$ brid Systems: Computation and Control, ser. Lecture Notes in Computer Science 2034. Springer-Verlag, 2001.

[5] C. Tomlin, G. J. Pappas, and S. Sastry, "Conflict resolution for air traffic management: A study in multiagent hybrid systems," IEEE Transactions on Automatic Control, vol. 43, no. 4, pp. 509-521, April 1998.

[6] J. Lygeros, D. N. Godbole, and S. Sastry, "Verified hybrid controllers for automated vehicles," IEEE Transactions on Automatic Control, vol. 43 , no. 4, pp. 522-539, April 1998.

[7] C. J. Tomlin, I. Mitchell, A. M. Bayen, and M. Oishi, "Computational techniques for the verification of hybrid systems," Proceedings of the IEEE, vol. 91, no. 7, pp. 986-1001, July 2003.

[8] W. H. Esselman, D. J. Sobajic, and J. Maulbetsch, "Hybrid discrete and continuous control for power systems," Discrete Event Dynamic Systems: Theory and Applications, vol. 9, pp. 297-318, 1999.

[9] L. H. Fink, "Discrete events in power systems," Discrete Event Dynamic Systems: Theory and Applications, vol. 9, pp. 319-330, 1999.

[10] I. A. Hiskens and M. A. Pai, "Hybrid systems view of power system modeling," in Proceedings of the IEEE International Symposium on Circuits and Systems, vol. II, Geneva, Switzerland, May 2000, pp. 228 231.

[11] T. A. Henzinger, "The theory of hybrid automata," in Proceedings of the 11th Annual IEEE Symposium on Logic in Computer Science, 1996, pp. 278-292.

[12] T. Sakiyama, T. Uemura, T. Ochi, T. Hikihara, Y. Susuki, and H. Ebina, "Application of hybrid system theory to power system analysis (IV)," in Annual Meeting Record I.E.E.Japan, vol. 6, 2006, pp. 269-270, (in Japanese).

[13] Y. Susuki, T. Hikihara, H. Ebina, T. Uemura, T. Ochi, and T. Sakiyama, "Application of hybrid system theory to power system analysis (V)," in Annual Meeting Record I.E.E.Japan, vol. 6, 2006, pp. 271-272, (in Japanese).

[14] T. Sakiyama, T. Uemura, T. Ochi, Y. Susuki, and T. Hikihara, "Study of transient stability evaluation method based on reachable sets: Evaluation of fault restoration method," in Proceedings of the Annual Conference of Power and Energy Society, IEEJ, vol. 6, 2006, p. 326, (in Japanese).

[15] I. M. Mitchell, "Comparing forward and backward reachability as tools for safety analysis," in Hybrid Systems: Computation and Control, ser. Lecture Notes in Computer Science 4416, A. Bemporad, A. Bicchi, and G. Buttazzo, Eds. Springer-Verlag, 2007, pp. 428-443.

[16] Y. Susuki, Y. Takatsuji, and T. Hikihara, "Hybrid dynamical system as model for cascading outage in a power system," in Proceedings of the 40th North American Power Symposium, Calgary, Canada, September 28-30 2008.
[17] T. A. Henzinger, P. H. Ho, and H. Wong-Toi, "Algorithmic analysis of nonlinear hybrid systems," IEEE Transactions on Automatic Control, vol. 43, no. 4, pp. 540-554, April 1998.

[18] M. R. Greenstreet and I. Mitchell, "Integrating projections," in Hybrid Systems: Computation and Control, ser. Lecture Notes in Computer Science 1386, T. A. Henzinger and S. Sastry, Eds. Springer Berlin / Heidelberg, 1998, pp. 159-174.

[19] H. D. Chiang, "Power system stability," in Wiley Encyclopedia of Electrical and Electronics Engineering, J. G. Webster, Ed. New York: John Wiley \& Sons, March 1999, pp. 105-137. 